\title{
NORMAL AUTOMORPHISMS OF A FREE METABELIAN NILPOTENT GROUP
}

\author{
GÉRARD ENDIMIONI \\ C.M.I-Université De Provence, 39, rue F. Joliot-Curie, F-13453 Marseille Cedex 13, France \\ e-mail: endimion@gyptis.univ-mrs.fr,Gerard.Endimioni@cmi.univ-mrs.fr
}

(Received 31 March 2008; revised 16 May 2009; accepted 21 September 2009)

\begin{abstract}
An automorphism $\varphi$ of a group $G$ is said to be normal if $\varphi(H)=H$ for each normal subgroup $H$ of $G$. These automorphisms form a group containing the group of inner automorphisms. When $G$ is a non-abelian free (or free soluble) group, it is known that these groups of automorphisms coincide, but this is not always true when $G$ is a free metabelian nilpotent group. The aim of this paper is to determine the group of normal automorphisms in this last case.
\end{abstract}

2000 Mathematics Subject Classification. 20E36, 20F28.

1. Preliminary results. In a group $G$, consider a $\operatorname{map} \varphi: G \rightarrow G$ of the form

$$
\varphi: x \mapsto x\left[x, u_{1}\right]^{\lambda(1)} \ldots\left[x, u_{m}\right]^{\lambda(m)},
$$

where $u_{1}, \ldots, u_{m}$ are elements of $G$, the exponents $\lambda(1), \ldots, \lambda(m)$ being integers (as usual, the commutator $[a, b]$ is defined by $\left.[a, b]=a^{-1} b^{-1} a b\right)$. When $G$ is metabelian, using the relation $[x y, u]=y^{-1}[x, u] y[y, u]$, it is easy to see that $\varphi$ is an endomorphism. These endomorphisms appear in [4] (also see [1]). Such endomorphisms are not necessarily automorphisms. But in a nilpotent group, each map of the form

$$
x \mapsto w_{0} x^{\lambda(1)} w_{1} x^{\lambda(2)} \ldots x^{\lambda(n)} w_{n} \quad(\text { with } \lambda(1)+\lambda(2)+\cdots+\lambda(n)= \pm 1)
$$

is bijective [2, Theorem 1]. Hence we have:

Proposition 1.1. In a metabelian nilpotent group $G$, every map $\varphi: G \rightarrow G$ of the form $\varphi: x \mapsto x \prod_{i=1}^{m}\left[x, u_{i}\right]^{\lambda(i)}\left(u_{i} \in G, \lambda(i) \in \mathbb{Z}\right)$ is an automorphism.

For convenience sake, in a metabelian nilpotent group, an automorphism of the form $x \mapsto x \prod_{i=1}^{m}\left[x, u_{i}\right]^{\lambda(i)}$ will be called a generalized inner automorphism.

As usual, in a group, the left-normed commutator $\left[x_{1}, \ldots, x_{n}\right]$ is defined inductively by

$$
\begin{aligned}
{\left[x_{1}, \ldots, x_{n}\right] } & =\left[x_{1}, \ldots, x_{n-1}\right]^{-1}\left[x_{1}, \ldots, x_{n-1}\right]^{x_{n}} \\
& =\left[x_{1}, \ldots, x_{n-1}\right]^{-1} x_{n}^{-1}\left[x_{1}, \ldots, x_{n-1}\right] x_{n} .
\end{aligned}
$$

The next technical result will be useful in the following. 
PROPOSITION 1.2. In a group $G$, consider a map $\varphi: G \rightarrow G$ of the form

$$
\varphi(x)=x \prod_{i=1}^{n}\left[x, v_{i, 1}, \ldots, v_{i, \sigma(i)}\right]^{\eta(i)} \quad(\eta(i) \in \mathbb{Z}),
$$

for some function $\sigma:\{1, \ldots, n\} \rightarrow \mathbb{N} \backslash\{0\}$ and elements $v_{i, j} \in G(1 \leq i \leq n, 1 \leq j \leq$ $\sigma(i))$. Then $\varphi(x)$ can be written in the form

$$
\varphi(x)=x\left[x, u_{1}\right]^{\lambda(1)} \ldots\left[x, u_{m}\right]^{\lambda(m)} \quad\left(\lambda(i) \in \mathbb{Z}, u_{i} \in G\right) .
$$

Proof. By induction, using the relation $[x, y, z]=[x, y]^{-1}[x, z]^{-1}[x, y z]$

Frequently in this paper we shall make use of well-known commutator identities (see for example [7, 5.1.5]). In particular, we have the following relations, valid in a metabelian group $G$, for any $x, y, z \in G, t \in G^{\prime}$ and $\lambda \in \mathbb{Z}$ :

$$
\begin{array}{cc}
{[x t, y]=[x, y][t, y],} & {\left[t^{\lambda}, y\right]=[t, y]^{\lambda},} \\
{[x, y, z][y, z, x][z, x, y]=1,} & {[t, x, y]=[t, y, x] .}
\end{array}
$$

PROPOSITION 1.3. The set of generalized inner automorphisms of a metabelian nilpotent group $G$ forms a (normal) subgroup of the group of automorphisms of $G$.

Proof. If $\varphi$ and $\psi$ are generalized inner automorphisms, the fact that $\psi \circ \varphi$ is a generalized inner automorphism follows from Proposition 1.2. It remains to prove that $\varphi^{-1}$ is a generalized inner automorphism. For that, it suffices to construct for each integer $k \geq 1$ a generalized inner automorphism $\psi_{k}$ such that $\psi_{k} \circ \varphi$ is of the form

$$
\psi_{k} \circ \varphi: x \mapsto x \prod_{i=1}^{m}\left[x, v_{i, 1}, \ldots, v_{i, \sigma(i)}\right]^{\eta(i)}
$$

for some function $\sigma:\{1, \ldots, m\} \rightarrow \mathbb{N} \backslash\{0\}$ and elements $v_{i, j} \in G(1 \leq i \leq m, 1 \leq j \leq$ $\sigma(i)$ ), and where each commutator is of weight $\geq 1+2^{k-1}$ (namely, $\sigma(i) \geq 2^{k-1}$ for $i=1, \ldots, m)$. Indeed, since $G$ is nilpotent, this implies that $\psi_{k} \circ \varphi(x)=x$ for $k$ large enough, thus $\varphi^{-1}=\psi_{k}$ is a generalized inner automorphism, as required. We argue by induction on $k$. The result is clear when $k=1$ by taking for $\psi_{1}$ the identity map. Now suppose that for some integer $k \geq 1$, there exists a generalized inner automorphism $\psi_{k}$ such that $\psi_{k} \circ \varphi(x)=x \prod_{i=1}^{m}\left[x, v_{i, 1}, \ldots, v_{i, \sigma(i)}\right]^{\eta(i)}$, with $\sigma(i) \geq 2^{k-1}$ for $i=1, \ldots, m$. Put $\psi_{k+1}=\psi^{\prime} \circ \psi_{k}$, where $\psi^{\prime}$ is defined by $\psi^{\prime}(x)=x \prod_{i=1}^{m}\left[x, v_{i, 1}, \ldots, v_{i, \sigma(i)}\right]^{-\eta(i)}$. We have

$$
\begin{aligned}
\psi_{k+1} \circ \varphi(x)= & x \prod_{i=1}^{m}\left[x, v_{i, 1}, \ldots, v_{i, \sigma(i)}\right]^{\eta(i)} \\
& \times \prod_{j=1}^{m}\left[x \prod_{i=1}^{m}\left[x, v_{i, 1}, \ldots, v_{i, \sigma(i)}\right]^{\eta(i)}, v_{j, 1}, \ldots, v_{j, \sigma(j)}\right]^{-\eta(j)} .
\end{aligned}
$$


Since

$$
\begin{aligned}
\prod_{j=1}^{m} & {\left[x \prod_{i=1}^{m}\left[x, v_{i, 1}, \ldots, v_{i, \sigma(i)}\right]^{\eta(i)}, v_{j, 1}, \ldots, v_{j, \sigma(j)}\right]^{-\eta(j)} } \\
& =\prod_{i=1}^{m}\left[x, v_{j, 1}, \ldots, v_{j, \sigma(j)}\right]^{-\eta(j)} \prod_{j=1}^{m} \prod_{i=1}^{m}\left[x, v_{i, 1}, \ldots, v_{i, \sigma(i)}, v_{j, 1}, \ldots, v_{j, \sigma(j)}\right]^{-\eta(i) \eta(j)},
\end{aligned}
$$

we obtain

$$
\psi_{k+1} \circ \varphi(x)=x \prod_{j=1}^{m} \prod_{i=1}^{m}\left[x, v_{i, 1}, \ldots, v_{i, \sigma(i)}, v_{j, 1}, \ldots, v_{j, \sigma(j)}\right]^{-\eta(i) \eta(j)}
$$

and this completes the proof of the proposition.

2. Main result. We recall that a normal automorphism $\varphi$ of a group $G$ is an automorphism such that $\varphi(H)=H$ for each normal subgroup $H$ of $G$. These automorphisms form a subgroup of the group of all automorphisms of $G$. Obviously, this subgroup contains the subgroup of inner automorphisms of $G$. It happens these subgroups coincide, for instance, when $G$ is a non-abelian free group [5], a non-abelian free soluble group [8], or a non-abelian free nilpotent group of class 2 [3]. On the other hand, the subgroup of inner automorphisms is of infinite index in the group of normal automorphisms when $G$ is a non-abelian free nilpotent group of class $k \geq 3$ [3]. Also note there are exactly two normal automorphisms in a (non-trivial) free abelian group: $x \mapsto x$ and $x \mapsto x^{-1}$.

Certainly, in a metabelian nilpotent group, each generalized inner automorphism is a normal automorphism, but a normal automorphism need not to be a generalized inner automorphism. However, our main result states that the converse holds in a non-abelian free metabelian nilpotent group.

THEOREM 2.1. In a non-abelian free metabelian nilpotent group, the group of normal automorphisms coincides with the group of generalized inner automorphisms.

3. Proof of Theorem 2.1. In all this section, we consider a fixed set $S$ of cardinality $\geq 2$ and we denote by $M_{k}$ the free metabelian nilpotent group of class $k>1$ freely generated by $S$. In other words, $M_{k}=F / F^{\prime \prime} \gamma_{k+1}(F)$, where $F$ is the free group freely generated by $S$ and $\gamma_{k+1}(F)$ the $(k+1)$ th term of the lower central series of $F$. The normal closure in a group $G$ of an element $a$ is written $\left\langle a^{G}\right\rangle$.

LEMMA 3.1. For any distinct elements $a, b \in S$ and any integer $t$, the subgroup $\left\langle\left(a^{t} b\right)^{M_{k}}\right\rangle \cap \gamma_{k}\left(M_{k}\right) \unlhd M_{k}$ is generated by the set of elements of the form $\left[a^{t} b, c_{1}, \ldots, c_{k-1}\right]$, with $c_{1}, \ldots, c_{k-1} \in S$.

Proof. First suppose that $t=0$ and consider an element $w \in\left\langle b^{M_{k}}\right\rangle \cap \gamma_{k}\left(M_{k}\right)$. Hence $w$ is a product of elements of the form $\left[c_{0}, c_{1}, \ldots, c_{k-1}\right]^{ \pm 1}$, with $c_{i} \in S$. More precisely, we can write $w=w_{0} w_{1}$, where $w_{0}$ (resp. $w_{1}$ ) is a product of elements of the form $\left[c_{0}, c_{1}, \ldots, c_{k-1}\right]^{ \pm 1}$ with $c_{i} \in S \backslash\{b\}$, (resp. with $c_{i} \in S$, the element $b$ occurring once at least in $\left.\left[c_{0}, c_{1}, \ldots, c_{k-1}\right]\right)$. In fact, substituting 1 for the indeterminate $b$ in the relation $w=w_{0} w_{1}$ and using the fact that $w$ lies in $\left\langle b^{M_{k}}\right\rangle$, we obtain $w_{0}=1$. 
Thus $w$ is a product of elements of the form $\left[c_{0}, c_{1}, \ldots, c_{k-1}\right]^{ \pm 1}$, with $c_{i}=b$ for some $i \in\{0, \ldots, k-1\}$. If $i=1$, we can write $\left[c_{0}, b, \ldots, c_{k-1}\right]=\left[b, c_{0}, \ldots, c_{k-1}\right]^{-1}$. If $i>1$, we have $\left[c_{0}, c_{1}, \ldots, b, \ldots, c_{k-1}\right]=\left[c_{0}, c_{1}, b, \ldots, c_{k-1}\right]$ and it follows:

$$
\left[c_{0}, c_{1}, \ldots, b, \ldots, c_{k-1}\right]=\left[b, c_{1}, c_{0}, \ldots, c_{k-1}\right]\left[b, c_{0}, c_{1}, \ldots, c_{k-1}\right]^{-1} .
$$

Thus we have shown that any element of $\left\langle b^{M_{k}}\right\rangle \cap \gamma_{k}\left(M_{k}\right)$ is a product of elements of the form $\left[b, c_{1}, \ldots, c_{k-1}\right]^{ \pm 1}$, with $c_{i} \in S$. Since $\left[b, c_{1}, \ldots, c_{k-1}\right] \in\left\langle b^{M_{k}}\right\rangle \cap \gamma_{k}\left(M_{k}\right)$, the lemma is proved when $t=0$.

Now consider the general case. Actually, since clearly $S^{\prime}=\left\{a^{t} b\right\} \cup S \backslash\{b\}$ is a free basis of $M_{k}$, we can use the result obtained in the particular case. It follows that $\left\langle\left(a^{t} b\right)^{M_{k}}\right\rangle \cap \gamma_{k}\left(M_{k}\right)$ is generated by the set of elements of the form $\left[a^{t} b, c_{1}, \ldots, c_{k-1}\right]$, with $c_{i} \in S^{\prime}$. But, in fact, we may take $c_{i} \in S$ and so conclude, since

$$
\left[a^{t} b, c_{1}, \ldots, a^{t} b, \ldots, c_{k-1}\right]=\left[a^{t} b, c_{1}, \ldots, a, \ldots, c_{k-1}\right]^{t}\left[a^{t} b, c_{1}, \ldots, b, \ldots, c_{k-1}\right] .
$$

As usual, the expression $[x, n y]$ is defined in a group by $[x, 0 y]=x$ and $[x, n y]=$ $[[x, n-1 y], y]$ for each positive integer $n$.

For a fixed subset $\left\{a_{0}, \ldots, a_{r}\right\} \subseteq S$ and a function $\Delta:\{0, \ldots, r\} \rightarrow \mathbb{N}$, we define in $M_{k}$ the symbol $[x, y, \Delta]\left(x, y \in M_{k}\right)$ by

$$
[x, y, \Delta]=\left[x, y,{ }_{\Delta(0)} a_{0}, \Delta(1) a_{1}, \ldots, \Delta(r) a_{r}\right] .
$$

Note that for any sequence $b_{1}, \ldots, b_{k}$ of elements of $\left\{a_{0}, \ldots, a_{r}\right\}$, there is a function $\Delta:\{0, \ldots, r\} \rightarrow \mathbb{N}$ such that $\left[x, y, b_{1}, \ldots, b_{k}\right]=[x, y, \Delta]$, with $\Delta(0)+\cdots+\Delta(r)=k$. If $j, j^{\prime}$ are distinct given integers in $\{0, \ldots, r\}$ and if $\Delta(j) \neq 0$, we define the function $\Delta_{(j)}^{\left(j^{\prime}\right)}:\{0, \ldots, r\} \rightarrow \mathbb{N}$ by

$$
\begin{aligned}
& \Delta_{(j)}^{\left(j^{\prime}\right)}(j)=\Delta(j)-1, \Delta_{(j)}^{\left(j^{\prime}\right)}\left(j^{\prime}\right)=\Delta\left(j^{\prime}\right)+1 \text { and } \\
& \Delta_{(j)}^{\left(j^{\prime}\right)}(i)=\Delta(i) \text { for all } i \in\{0, \ldots, r\} \backslash\left\{j, j^{\prime}\right\} .
\end{aligned}
$$

When $\Delta$ is not the zero-function, we shall denote by $m(\Delta)$ the least integer $j$ such that $\Delta(j) \neq 0$.

If $S$ is ordered, we may define in $M_{k}$ basic commutators (see for example [6, Chapter 3]). Recall that a basic commutator of weight $k^{\prime}\left(2 \leq k^{\prime} \leq k\right)$ is a commutator of the form $\left[b_{1}, b_{2}, \ldots, b_{k^{\prime}}\right]\left(b_{i} \in S\right)$, with $b_{1}>b_{2}$ and $b_{2} \leq b_{3} \leq \cdots \leq b_{k^{\prime}}$. Any set of these commutators freely generates a free abelian subgroup of $\bar{M}_{k}^{\prime}$.

In the next lemma, we aim to express a product of commutators of the form $\left[a_{s}, a_{i}, \Delta\right]$ as a product where only basic commutators occur.

LeMmA 3.2. Let $\left\{a_{0}, \ldots, a_{r}\right\}$ be a finite subset of $S(r>0)$. Choose an integer $s \in\{0, \ldots, r\}$ and consider an element $w \in M_{k+2}(k>0)$ of the form

$$
w=\prod_{i, \Delta}\left[a_{s}, a_{i}, \Delta\right]^{\epsilon(i, \Delta)} \quad(\epsilon(i, \Delta) \in \mathbb{Z}),
$$

where the product is taken over all integers $i \in\{0, \ldots, r\} \backslash\{s\}$ and all functions $\Delta$ : $\{0, \ldots, r\} \rightarrow \mathbb{N}$ such that $\Delta(0)+\cdots+\Delta(r)=k$. Then: 
(i) We have

$$
\begin{aligned}
w= & \prod_{i<s, i \leq m(\Delta)}\left[a_{s}, a_{i}, \Delta\right]^{\epsilon(i, \Delta)} \prod_{s<i, s \leq m(\Delta)}\left[a_{i}, a_{s}, \Delta\right]^{-\epsilon(i, \Delta)} \\
& \times \prod_{m(\Delta)<s, m(\Delta)<i}\left[a_{i}, a_{m(\Delta)}, \Delta_{(m(\Delta))}^{(s)}\right]^{-\epsilon(i, \Delta)} \prod_{m(\Delta)<s, m(\Delta)<i}\left[a_{s}, a_{m(\Delta)}, \Delta_{(m(\Delta))}^{(i)}\right]^{\epsilon(i, \Delta)}
\end{aligned}
$$

(in all these products, $i$ lies in $\{0, \ldots, r\} \backslash\{s\}$ ).

(ii) We have $w=1$ only if all exponents $\epsilon(i, \Delta)$ with $i \in\{0, \ldots, r\} \backslash\{s\}$ occurring in the expression of $w$ are zero.

Proof. (i) First we write $w$ as a product of two factors:

$$
w=\prod_{i \leq m(\Delta)}\left[a_{s}, a_{i}, \Delta\right]^{\epsilon(i, \Delta)} \prod_{m(\Delta)<i}\left[a_{s}, a_{i}, \Delta\right]^{\epsilon(i, \Delta)} .
$$

The first factor can be expressed in the form

$$
\begin{aligned}
\prod_{i \leq m(\Delta)}\left[a_{s}, a_{i}, \Delta\right]^{\epsilon(i, \Delta)} & =\prod_{i<s, i \leq m(\Delta)}\left[a_{s}, a_{i}, \Delta\right]^{\epsilon(i, \Delta)} \prod_{s<i \leq m(\Delta)}\left[a_{s}, a_{i}, \Delta\right]^{\epsilon(i, \Delta)} \\
& =\prod_{i<s, i \leq m(\Delta)}\left[a_{s}, a_{i}, \Delta\right]^{\epsilon(i, \Delta)} \prod_{s<i \leq m(\Delta)}\left[a_{i}, a_{s}, \Delta\right]^{-\epsilon(i, \Delta)} .
\end{aligned}
$$

In the same way, we have

$$
\begin{aligned}
\prod_{m(\Delta)<i}\left[a_{s}, a_{i}, \Delta\right]^{\epsilon(i, \Delta)} & =\prod_{s \leq m(\Delta)<i}\left[a_{s}, a_{i}, \Delta\right]^{\epsilon(i, \Delta)} \prod_{m(\Delta)<s, m(\Delta)<i}\left[a_{s}, a_{i}, \Delta\right]^{\epsilon(i, \Delta)} \\
& =\prod_{s \leq m(\Delta)<i}\left[a_{i}, a_{s}, \Delta\right]^{-\epsilon(i, \Delta)} \prod_{m(\Delta)<s, m(\Delta)<i}\left[a_{s}, a_{i}, \Delta\right]^{\epsilon(i, \Delta)}
\end{aligned}
$$

Therefore Lemma 3.2(i) is proved if we show the relation

$$
\begin{aligned}
\prod_{m(\Delta)<s, m(\Delta)<i}\left[a_{s}, a_{i}, \Delta\right]^{\epsilon(i, \Delta)}= & \prod_{m(\Delta)<s, m(\Delta)<i}\left[a_{i}, a_{m(\Delta)}, \Delta_{(m(\Delta))}^{(s)}\right]^{-\epsilon(i, \Delta)} \\
& \times \prod_{m(\Delta)<s, m(\Delta)<i}\left[a_{s}, a_{m(\Delta)}, \Delta_{(m(\Delta))}^{(i)}\right]^{\epsilon(i, \Delta)} .
\end{aligned}
$$

For that, write more explicitly the commutator $\left[a_{s}, a_{i}, \Delta\right]$ (in the following equalities, we write $m$ instead of $m(\Delta)$ ):

$$
\begin{aligned}
{\left[a_{s}, a_{i}, \Delta\right] } & =\left[a_{s}, a_{i}, \Delta(0) a_{0}, \ldots, \Delta(r) a_{r}\right] \\
& =\left[a_{s}, a_{i}, \Delta(m) a_{m}, \ldots, \Delta(r) a_{r}\right] \\
& =\left[a_{s}, a_{i}, a_{m}, \Delta(m)-1 a_{m}, \ldots, \Delta(r) a_{r}\right] .
\end{aligned}
$$

Since $\left[a_{s}, a_{i}, a_{m}\right]=\left[a_{i}, a_{m}, a_{s}\right]^{-1}\left[a_{m}, a_{s}, a_{i}\right]^{-1}=\left[a_{i}, a_{m}, a_{s}\right]^{-1}\left[a_{s}, a_{m}, a_{i}\right]$, we obtain

$$
\left[a_{s}, a_{i}, \Delta\right]=\left[a_{i}, a_{m}, \Delta_{(m)}^{(s)}\right]^{-1}\left[a_{s}, a_{m}, \Delta_{(m)}^{(i)}\right] .
$$

Relation (1) is now an immediate consequence of (2). 
(ii) Choose an ordering of $S$ such that $a_{s}$ is the lowest element. Then, since $\left[a_{s}, a_{i}, \Delta\right]=$ $\left[a_{i}, a_{s}, \Delta\right]^{-1}$, all commutators involved in $w$ are inverses of basic commutators, and the basic commutators that occur are distinct. The result follows.

LEMMA 3.3. Let $\varphi$ be a normal automorphism of $M_{k+2}(k>0)$ acting trivially on $M_{k+2} / \gamma_{k+2}\left(M_{k+2}\right)$. Then, for all distinct elements $a, b \in S$, there exists a generalized inner automorphism $\psi$ of $M_{k+2}$ such that $\varphi(a)=\psi(a)$ and $\varphi(b)=\psi(b)$.

Proof. Let $a, b$ be two distinct elements of $S$. Then $a^{-1} \varphi(a)$ and $b^{-1} \varphi(b)$ belong to $\left\langle a^{M_{k+2}}\right\rangle \cap \gamma_{k+2}\left(M_{k+2}\right)$ and $\left\langle b^{M_{k+2}}\right\rangle \cap \gamma_{k+2}\left(M_{k+2}\right)$ respectively. By Lemma 3.1, there is a finite subset $\left\{a=a_{0}, a_{1}, \ldots, a_{r}=b\right\} \subseteq S$ such that

$$
\begin{aligned}
& \varphi(a)=\varphi\left(a_{0}\right)=a_{0} \prod_{i, \Delta}\left[a_{0}, a_{i}, \Delta\right]^{\alpha(i, \Delta)} \quad(\alpha(i, \Delta) \in \mathbb{Z}), \\
& \varphi(b)=\varphi\left(a_{r}\right)=a_{r} \prod_{i, \Delta}\left[a_{r}, a_{i}, \Delta\right]^{\beta(i, \Delta)} \quad(\beta(i, \Delta) \in \mathbb{Z}),
\end{aligned}
$$

where the two products are taken over all integers $i \in\{0, \ldots, r\}$ and all functions $\Delta$ : $\{0, \ldots, r\} \rightarrow \mathbb{N}$ with $\Delta(0)+\cdots+\Delta(r)=k$ (as in Lemma 3.2, $\left[a_{s}, a_{i}, \Delta\right]$ is defined by $\left.\left[a_{s}, a_{i}, \Delta\right]=\left[a_{s}, a_{i}, \Delta(0) a_{0}, \ldots, \Delta(r) a_{r}\right]\right)$. Note that if $|S|=2$ (and so $r=1$ ), Lemma 3.3 is easily verified by taking the generalized inner automorphism $\psi$ defined by

$$
\psi(x)=x \prod_{\Delta}\left[x, a_{1}, \Delta\right]^{\alpha(1, \Delta)} \prod_{\Delta}\left[x, a_{0}, \Delta\right]^{\beta(0, \Delta)} .
$$

Thus we shall assume in the following that $|S|>2$. By Lemma 3.1, for any positive integer $t,\left(a_{0}^{t} a_{r}\right)^{-1} \varphi\left(a_{0}^{t} a_{r}\right)$ can be expressed as a product of elements of the form $\left[a_{0}^{t} a_{r}, c_{1}, \ldots, c_{k+1}\right]^{ \pm 1}$, with $c_{1}, \ldots, c_{k+1} \in S$. But $\left(a_{0}^{t} a_{r}\right)^{-1} \varphi\left(a_{0}^{t} a_{r}\right)=\left(a_{0}^{t} a_{r}\right)^{-1} \varphi\left(a_{0}\right)^{t} \varphi\left(a_{r}\right)$ belongs to $\left\langle a_{0}, a_{1}, \ldots, a_{r}\right\rangle$. Therefore, substituting 1 for all indeterminates in $S \backslash$ $\left\{a_{0}, a_{1}, \ldots, a_{r}\right\}$ in the expression of $\left(a_{0}^{t} a_{r}\right)^{-1} \varphi\left(a_{0}^{t} a_{r}\right)$, we can assume that $c_{1}, \ldots, c_{k+1} \in$ $\left\{a_{0}, a_{1}, \ldots, a_{r}\right\}$. It follows that $\varphi\left(a_{0}^{t} a_{r}\right)$ can be expressed in the form

$$
\begin{aligned}
\varphi\left(a_{0}^{t} a_{r}\right) & =a_{0}^{t} a_{r} \prod_{i, \Delta}\left[a_{0}^{t} a_{r}, a_{i}, \Delta\right]^{\eta_{t}(i, \Delta)} \quad\left(\eta_{t}(i, \Delta) \in \mathbb{Z}\right) \\
& =a_{0}^{t} a_{r} \prod_{i, \Delta}\left[a_{0}, a_{i}, \Delta\right]^{t \eta_{t}(i, \Delta)} \prod_{i, \Delta}\left[a_{r}, a_{i}, \Delta\right]^{\eta_{t}(i, \Delta)} .
\end{aligned}
$$

Thus the relation $\varphi\left(a_{0}^{t} a_{r}\right)=\varphi\left(a_{0}\right)^{t} \varphi\left(a_{r}\right)$ implies

$$
\begin{aligned}
& \prod_{i, \Delta}\left[a_{0}, a_{i}, \Delta\right]^{t \eta_{t}(i, \Delta)} \prod_{i, \Delta}\left[a_{r}, a_{i}, \Delta\right]^{\eta_{t}(i, \Delta)} \\
& =\prod_{i, \Delta}\left[a_{0}, a_{i}, \Delta\right]^{t \alpha(i, \Delta)} \prod_{i, \Delta}\left[a_{r}, a_{i}, \Delta\right]^{\beta(i, \Delta)} .
\end{aligned}
$$

Choose an order in $S$ such that $a_{0}<a_{1}<\cdots<a_{r}$ and express each product in (3) as a product of basic commutators (or their inverses). Considering, for instance, the left-hand side of (3) (the treatment of the righthand side is similar), we have

$$
\prod_{i, \Delta}\left[a_{0}, a_{i}, \Delta\right]^{t \eta_{t}(i, \Delta)}=\prod_{i \neq 0, \Delta}\left[a_{i}, a_{0}, \Delta\right]^{-t \eta_{t}(i, \Delta)}
$$


and, by using Lemma 3.2(i) with $s=r$,

$$
\begin{aligned}
\prod_{i, \Delta}\left[a_{r}, a_{i}, \Delta\right]^{\eta_{t}(i, \Delta)}= & \prod_{i \neq r, i \leq m(\Delta)}\left[a_{r}, a_{i}, \Delta\right]^{\eta_{t}(i, \Delta)} \\
& \times \prod_{i \neq r, m(\Delta)<i}\left[a_{i}, a_{m(\Delta)}, \Delta_{(m(\Delta))}^{(r)}\right]^{-\eta_{t}(i, \Delta)} \prod_{i \neq r, m(\Delta)<i}\left[a_{r}, a_{m(\Delta)}, \Delta_{(m(\Delta))}^{(i)}\right]^{\eta_{t}(i, \Delta)} .
\end{aligned}
$$

Thus relation (3) can be written in the form

$$
\begin{aligned}
& \prod_{i \neq 0, \Delta}\left[a_{i}, a_{0}, \Delta\right]^{-t \eta_{t}(i, \Delta)} \prod_{i \neq r, i \leq m(\Delta)}\left[a_{r}, a_{i}, \Delta\right]^{\eta_{t}(i, \Delta)} \\
& \quad \times \prod_{i \neq r, m(\Delta)<i}\left[a_{i}, a_{m(\Delta)}, \Delta_{(m(\Delta))}^{(r)}\right]^{-\eta_{t}(i, \Delta)} \prod_{i \neq r, m(\Delta)<i}\left[a_{r}, a_{m(\Delta)}, \Delta_{(m(\Delta))}^{(i)}\right]^{\eta_{t}(i, \Delta)} \\
& =\prod_{i \neq 0, \Delta}\left[a_{i}, a_{0}, \Delta\right]^{-t \alpha(i, \Delta)} \prod_{i \neq r, i \leq m(\Delta)}\left[a_{r}, a_{i}, \Delta\right]^{\beta(i, \Delta)} \\
& \quad \times \prod_{i \neq r, m(\Delta)<i}\left[a_{i}, a_{m(\Delta)}, \Delta_{(m(\Delta))}^{(r)}\right]^{-\beta(i, \Delta)} \prod_{i \neq r, m(\Delta)<i}\left[a_{r}, a_{m(\Delta)}, \Delta_{(m(\Delta))}^{(i)}\right]^{\beta(i, \Delta)} .
\end{aligned}
$$

Now consider an integer $i \in\{1, \ldots, r-1\}$ and a function $\Delta:\{0, \ldots, r\} \rightarrow \mathbb{N}$, with $\Delta(0)+\cdots+\Delta(r)=k$ (we can always suppose that $r>1$ since $|S|>2$ ). By identifying the exponents of the basic commutator $\left[a_{i}, a_{0}, \Delta\right]$ of each side of relation (4), it is easy to see that

$$
t \eta_{t}(i, \Delta)+\eta_{t}\left(i, \Delta_{(r)}^{(0)}\right)=t \alpha(i, \Delta)+\beta\left(i, \Delta_{(r)}^{(0)}\right)
$$

if $\Delta(r)>0$, and $\eta_{t}(i, \Delta)=\alpha(i, \Delta)$ if $\Delta(r)=0$. We prove by induction on $\Delta(r)$ that actually, we have always the equality $\eta_{t}(i, \Delta)=\alpha(i, \Delta)$. At first observe that if $\Delta(r)>0$, we have $\Delta_{(r)}^{(0)}(r)=\Delta(r)-1$ and so $\eta_{t}\left(i, \Delta_{(r)}^{(0)}\right)=\alpha\left(i, \Delta_{(r)}^{(0)}\right)$ by induction. Hence relation (5) implies

$$
\alpha\left(i, \Delta_{(r)}^{(0)}\right)-\beta\left(i, \Delta_{(r)}^{(0)}\right)=t\left\{\alpha(i, \Delta)-\eta_{t}(i, \Delta)\right\}
$$

Consequently, each positive integer $t$ divides the integer $\alpha\left(i, \Delta_{(r)}^{(0)}\right)-\beta\left(i, \Delta_{(r)}^{(0)}\right)$, which is independent of $t$. It follows that $\alpha\left(i, \Delta_{(r)}^{(0)}\right)=\beta\left(i, \Delta_{(r)}^{(0)}\right)$ and so $\eta_{t}(i, \Delta)=\alpha(i, \Delta)$, as required.

Using theses relations and taking $t=1$, relation (3) implies

$$
\prod_{\Delta}\left[a_{0}, a_{r}, \Delta\right]^{\eta(r, \Delta)} \prod_{i, \Delta}\left[a_{r}, a_{i}, \Delta\right]^{\eta(i, \Delta)}=\prod_{\Delta}\left[a_{0}, a_{r}, \Delta\right]^{\alpha(r, \Delta)} \prod_{i, \Delta}\left[a_{r}, a_{i}, \Delta\right]^{\beta(i, \Delta)}
$$

(we write $\eta$ for $\eta_{1}$ ) and so

$$
\prod_{i, \Delta}\left[a_{r}, a_{i}, \Delta\right]^{\beta(i, \Delta)}=\prod_{i, \Delta}\left[a_{r}, a_{i}, \Delta\right]^{\eta(i, \Delta)} \prod_{\Delta}\left[a_{r}, a_{0}, \Delta\right]^{\alpha(r, \Delta)-\eta(r, \Delta)} .
$$

Since $\varphi\left(a_{r}\right)=a_{r} \prod_{i, \Delta}\left[a_{r}, a_{i}, \Delta\right]^{\beta(i, \Delta)}$, we obtain

$$
\varphi\left(a_{r}\right)=a_{r} \prod_{i, \Delta}\left[a_{r}, a_{i}, \Delta\right]^{\eta(i, \Delta)} \prod_{\Delta}\left[a_{r}, a_{0}, \Delta\right]^{\alpha(r, \Delta)-\eta(r, \Delta)}
$$


Now consider the generalized inner automorphism $\psi$ defined by

$$
\psi(x)=x \prod_{i=1, \ldots, r, \Delta}\left[x, a_{i}, \Delta\right]^{\alpha(i, \Delta)} \prod_{\Delta}\left[x, a_{0}, \Delta\right]^{\alpha(r, \Delta)+\eta(0, \Delta)-\eta(r, \Delta)} .
$$

We have

$$
\psi\left(a_{0}\right)=a_{0} \prod_{i=1, \ldots, r, \Delta}\left[a_{0}, a_{i}, \Delta\right]^{\alpha(i, \Delta)}=\varphi\left(a_{0}\right)
$$

In the same way,

$$
\begin{aligned}
\psi\left(a_{r}\right) & =a_{r} \prod_{i=1, \ldots, r-1, \Delta}\left[a_{r}, a_{i}, \Delta\right]^{\alpha(i, \Delta)} \prod_{\Delta}\left[a_{r}, a_{0}, \Delta\right]^{\alpha(r, \Delta)+\eta(0, \Delta)-\eta(r, \Delta)} \\
& =a_{r} \prod_{i=1, \ldots, r-1, \Delta}\left[a_{r}, a_{i}, \Delta\right]^{\eta(i, \Delta)} \prod_{\Delta}\left[a_{r}, a_{0}, \Delta\right]^{\alpha(r, \Delta)+\eta(0, \Delta)-\eta(r, \Delta)} \\
& =a_{r} \prod_{i, \Delta}\left[a_{r}, a_{i}, \Delta\right]^{\eta(i, \Delta)} \prod_{\Delta}\left[a_{r}, a_{0}, \Delta\right]^{\alpha(r, \Delta)-\eta(r, \Delta)}
\end{aligned}
$$

and so $\psi\left(a_{r}\right)=\varphi\left(a_{r}\right)$ by (6). This completes the proof of Lemma 3.3.

LEMMA 3.4. Let $\varphi$ be a normal automorphism of $M_{k+2}(k>0)$ acting trivially on $M_{k+2} / \gamma_{k+2}\left(M_{k+2}\right)$. Then $\varphi$ is a generalized inner automorphism of $M_{k+2}$.

Proof. We can assume that $|S|>2$ (otherwise Lemma 3.4 is a consequence of Lemma 3.3). Consider two distinct elements $a, b \in S$. According to Lemma 3.3, there exists a generalized inner automorphism $\psi$ such that $\varphi(a)=\psi(a)$ and $\varphi(b)=\psi(b)$. It suffices to prove that for any $c \in S \backslash\{a, b\}$, we have $\varphi(c)=\psi(c)$. For that, apply again Lemma 3.3: there are generalized inner automorphisms $\psi^{\prime}, \psi^{\prime \prime}$ such that $\varphi(a)=\psi^{\prime}(a)$, $\varphi(c)=\psi^{\prime}(c)$ and $\varphi(b)=\psi^{\prime \prime}(b), \varphi(c)=\psi^{\prime \prime}(c)$. There exists a finite subset $\left\{a_{0}, \ldots, a_{r}\right\} \subseteq$ $S$, containing $a, b, c$, such that $\psi, \psi^{\prime}, \psi^{\prime \prime}$ can be defined by the equations

$$
\begin{aligned}
\psi(x) & =x \prod_{i, \Delta}\left[x, a_{i}, \Delta\right]^{\epsilon(i, \Delta)} \\
\psi^{\prime}(x) & =x \prod_{i, \Delta}\left[x, a_{i}, \Delta\right]^{\epsilon^{\prime}(i, \Delta)} \\
\psi^{\prime \prime}(x) & =x \prod_{i, \Delta}\left[x, a_{i}, \Delta\right]^{\epsilon^{\prime \prime}(i, \Delta)}
\end{aligned}
$$

(the products are taken over all integers $i \in\{0, \ldots, r\}$ and all functions $\Delta:\{0, \ldots, r\} \rightarrow$ $\mathbb{N}$ with $\Delta(0)+\cdots+\Delta(r)=k)$. Since $\psi(a)=\psi^{\prime}(a)$, we have

$$
a \prod_{i, \Delta}\left[a, a_{i}, \Delta\right]^{\epsilon(i, \Delta)}=a \prod_{i, \Delta}\left[a, a_{i}, \Delta\right]^{\epsilon^{\prime}(i, \Delta)}
$$

and so

$$
\prod_{i, \Delta}\left[a, a_{i}, \Delta\right]^{\epsilon(i, \Delta)-\epsilon^{\prime}(i, \Delta)}=1 .
$$

Applying Lemma 3.2(ii), we obtain $\epsilon(i, \Delta)=\epsilon^{\prime}(i, \Delta)$ for all functions $\Delta$ and all integers $i \in\{0, \ldots, r\}$ such that $a_{i} \neq a$. Similarly, we have $\epsilon(i, \Delta)=\epsilon^{\prime \prime}(i, \Delta)$ if $a_{i} \neq b$ 
and $\epsilon^{\prime}(i, \Delta)=\epsilon^{\prime \prime}(i, \Delta)$ if $a_{i} \neq c$. It follows that $\epsilon(i, \Delta)=\epsilon^{\prime}(i, \Delta)$ for all function $\Delta$ and all integer $i \in\{0, \ldots, r\}$, hence $\psi=\psi^{\prime}$. Thus $\varphi(c)=\psi^{\prime}(c)=\psi(c)$, as required.

Proof of Theorem 2.1. We argue by induction on the nilpotency class $k$ of $M_{k}$. If $k=$ 2, the result follows from [3, Theorem 2(ii)] (in this case, each normal automorphism is inner). Now consider a normal automorphism $\varphi$ of $M_{k}$, with $k>2$. Then $\varphi$ induces a normal automorphism on the quotient group $M_{k} / \gamma_{k}\left(M_{k}\right)$. By induction, since this quotient is isomorphic to $M_{k-1}$, there exists a generalized inner automorphism $\psi$ : $M_{k} \rightarrow M_{k}$ such that $\varphi(x)=\psi(x) \theta(x)$, where $\theta(x)$ is an element of $\gamma_{k}\left(M_{k}\right)$. It follows that $\psi^{-1}(\varphi(x))=x \psi^{-1}(\theta(x))$. Thus $\psi^{\prime}:=\psi^{-1} \circ \varphi$ is a normal automorphism of $M_{k}$ acting trivially on $M_{k} / \gamma_{k}\left(M_{k}\right)$. By Lemma 3.4, $\psi^{\prime}$ is a generalized inner automorphism, and so is $\varphi=\psi \circ \psi^{\prime}$. This completes the proof of Theorem 2.1.

\section{REFERENCES}

1. A. Caranti and C. M. Scoppola, Endomorphisms of two-generated metabelian groups that induce the identity modulo the derived subgroup, Arch. Math. 56 (1991), 218-227.

2. G. Endimioni, Applications rationnelles d'un groupe nilpotent, C. R. Acad. Sci. Paris 314 (1992), 431-434.

3. G. Endimioni, Pointwise inner automorphisms in a free nilpotent group, Quart. J. Math. 53 (2002), 397-402.

4. J. V. Kuz'min, Inner endomorphisms of metabelian groups (Russian), Sibirsk. Mat. Zh. 16 (1975), 736-744; Translation in Siberian Math. J. 16 (1976), 563-568.

5. A. Lubotzky, Normal automorphisms of free groups, J. Algebra 63 (1980), 494-498.

6. H. Neumann, Varieties of groups (Springer-Verlag, Berlin, 1967).

7. D. J. S. Robinson, A course in the theory of groups (Springer-Verlag, New York, 1996).

8. N. S. Romanovskiı̌, Normal automorphisms of free solvable pro-p-groups (Russian). Algebra i Logika 36 (1997), 441-453; Translation in Algebra and Logic 36 (1997), 257-263. 